Research Article

\title{
Nuclear and Detector Sensitivities for Neutrinoless Double Beta-Decay Experiments
}

\author{
Hiroyasu Ejiri (iD \\ Research Center for Nuclear Physics, Osaka University, Osaka 567-0047, Japan \\ Correspondence should be addressed to Hiroyasu Ejiri; ejiri@rcnp.osaka-u.ac.jp
}

Received 7 December 2020; Accepted 15 January 2021; Published 27 January 2021

Academic Editor: Antonio J. Accioly

Copyright @ 2021 Hiroyasu Ejiri. This is an open access article distributed under the Creative Commons Attribution License, which permits unrestricted use, distribution, and reproduction in any medium, provided the original work is properly cited. The publication of this article was funded by SCOAP ${ }^{3}$.

\begin{abstract}
Neutrinoless double beta-decay (DBD) is of current interest in high-sensitivity frontiers of particle physics. The decay is very sensitive to Majorana neutrino masses, neutrino CP phases, right-handed weak interactions, and others, which are beyond the standard electroweak model. DBDs are actually ultrarare events, and thus, DBD experiments with ultrahigh sensitivity are required. Critical discussions are presented on nuclear and detector sensitivities for high-sensitivity DBD experiments to study the neutrino masses in the normal and inverted mass hierarchies.
\end{abstract}

\section{Introduction}

Neutrinoless double beta-decay (DBD) is of current interest in sensitivity frontiers of particle physics. The decay violates the lepton number conservation law, and thus, DBD is beyond the standard electroweak model. DBD is very sensitive to the Majorana nature (neutrino=antineutrino) of neutrino, the absolute neutrino mass scale and the neutrino mass hierarchy, the neutrino $\mathrm{CP}$ phases, the possible righthanded weak interactions, and others, which are beyond the standard model. Accordingly, it has been used to study these fundamental questions on the neutrino and the weak interaction for decades. Recent experimental and theoretical DBD works are discussed in the review articles and references therein [1-5].

In fact, neutrinoless DBDs are due to various modes such as the light neutrino mass mode, the right-handed weak current mode, and the SUSY particle exchange mode, which are all beyond the standard model $[1,4]$. In the present work, we discuss mainly the neutrinoless DBD due to the light neutrino mass mode, which is of current interest.

DBD, however, is a very low-energy and ultrarare decay. The energy is of the order of $E_{\beta \beta} \approx 10^{-3} \mathrm{GeV}$, and the decay rate is in the range of $T_{r}^{0 v} \approx 10^{-27}-10^{-30}$ per year (y), depending on the neutrino mass and the nuclear matrix element
(NME). The neutrino mass to be studied is around $m=3$ $\mathrm{meV}$ and $30 \mathrm{meV}$ in cases of the normal mass hierarchy $(\mathrm{NH})$ and the inverted mass hierarchy, respectively. Then, low-background high-sensitivity DBD detectors are required to search for such low-energy and ultrarare DBD signals $[1,4]$.

Many experimental groups are working hard for highsensitivity DBD experiments to search for the small Majorana neutrino mass and others beyond the standard model. Current lower limits on the DBD half-lives are of the order of $10^{26} \mathrm{y}$ for both ${ }^{76} \mathrm{Ge}$ and ${ }^{136} \mathrm{Xe}$. The neutrino mass limits are, respectively, $600 \mathrm{meV} / M(76)$ and $230 \mathrm{meV} / M(136)$ with $M(76)$ and $M(136)$ being the nuclear matrix elements (NMEs) for ${ }^{76} \mathrm{Ge}$ and ${ }^{136} \mathrm{Xe}$, respectively. So, they are in the range of 300-100 meV if NMEs are around 2 (see Section 4 for discussions on DBD experiments). Then, one needs to improve the sensitivity by factors 5 and 50 to access the $\mathrm{IH}$ and $\mathrm{NH}$ mass regions, respectively. In fact, the DBD sensitivity to the neutrino mass depends much on NME.

DBD with a light neutrino mass mode is considered a neutrino exchange process, where a light Majorana neutrino is exchanged between two neutrons in the DBD nucleus. Here, the nucleus acts as a high-luminosity microcollider of two neutrons, as shown in Figure 1. The neutrino exchange cross-section for the IH neutrino is as small as $\sigma \approx 10^{-83}$ $\mathrm{cm}^{2}$ because of the second-order weak process and the small 
Femto $\left(10^{-15} \mathrm{~m}\right)$ nuclear collider $\quad$ Femto $\left(10^{-15} \mathrm{~m}\right)$ nuclear collider

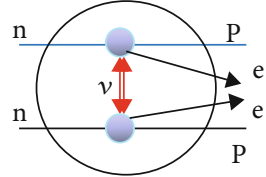

Double beta decay

(a)

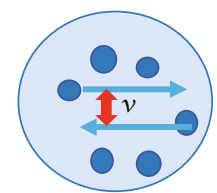

Nuclear collider

(b)
Figure 1: Schematic views of neutrinoless double $\beta$-decay with Majorana neutrino exchange (a) and nuclear collider (b).

neutrino mass $[4,6,7]$. The luminosity of one nucleus is as high as $L \approx 10^{48} \mathrm{~cm}^{-2} \mathrm{~s}^{-1}$ because of the very small (a few $\mathrm{fm}$ ) distance between the two neutrons in the nucleus. The summed luminosity for 1 ton DBD nuclei is $L \approx 10^{76} \mathrm{~cm}^{-2}$ $\mathrm{s}^{-1}$. Thus, one gets 2-3 DBD signals per year for the IH neutrino exchange by using a large DBD detector with 1 ton DBD isotopes.

The DBD neutrino mass sensitivity to search for the small neutrino mass is defined as the minimum neutrino mass $m_{m}$ to be measured by the DBD experiment. It is given by a product of a nuclear sensitivity and a detector sensitivity. The nuclear sensitivity depends on nuclear parameters such as the nuclear phase space and the NME, while the detector sensitivity depends on the detector parameters such as the total number of the DBD isotopes, the enrichment, the exposure time, the detection efficiency, and the backgrounds. The minimum neutrino mass to be measured and the nuclear and detector sensitivities are discussed in the review articles $[1,4,7]$.

The present report is aimed at critically discussing the nuclear and detector sensitivities to search for the ultrarare DBD events associated with very small $\mathrm{IH}$ and $\mathrm{NH}$ masses. Critical discussions on DBD NMEs are presented elsewhere [8].

\section{Neutrino Mass Sensitivity for DBD Experiment}

The neutrinoless DBD transition rate is expressed in terms of the axial-vector weak coupling $g_{A}=1.27$ in units of the vector coupling of $g_{V}$ as $[1,4]$

$$
T_{r}^{0 v}=g_{A}^{4} G^{0 v}\left|M^{0 v} f(v)\right|^{2}
$$

where $G^{0 v}$ is the phase space volume, $M^{0 v}$ is the NME, and $f(v)$ stands for the effective neutrino mass and the other neutrino and weak interaction parameters beyond the standard model. These three quantities are associated with the kinematic factor, the nuclear physics factor, and the particle physics factor, respectively.

In case of the light neutrino mass process, $f(v)$ is given by the effective neutrino mass of $m=\sum \bigotimes\left|U_{i}\right|^{2} m_{i} e^{\alpha_{i}}$ with $m_{i}, U_{i}$, and $\alpha_{i}$ being the $i$ th neutrino mass, the mixing coefficient, and the phase, respectively. In case of the right-handed weak-boson process, $f(v)$ includes the term $k\left(M_{W}^{L} / M_{W}^{R}\right)^{2}$ with $M_{W}^{L}$ and $M_{W}^{R}$ being the left-handed and right-handed weak-boson masses, respectively $[1,4]$.

The DBD NME for the light neutrino mass mode is expressed in terms of the Gamow-Teller (GT), the Fermi $(\mathrm{F})$, and the tensor (T) NMEs as $[1,4,7]$

$$
M^{0 v}=\left(\frac{g_{A}^{\mathrm{eff}}}{g_{A}}\right)^{2}\left[M(\mathrm{GT})+\left(\frac{g_{V}^{\mathrm{eff}}}{g_{A}^{\mathrm{eff}}}\right)^{2} M(\mathrm{~F})+M(\mathrm{~T})\right],
$$

where $g_{A}^{\text {eff }}$ and $g_{V}^{\text {eff }}$ are, respectively, the effective axial-vector and vector couplings and $g_{A}$ and $g_{V}$ are the axial-vector and vector couplings for a free nucleon. $M(\mathrm{GT}), M(\mathrm{~F})$, and $M(\mathrm{~T})$ are, respectively, the model NMEs for Gamow-Teller, Fermi, and tensor transition operators.

The GT and T NMEs are very sensitive to nucleonic and nonnucleonic correlations and nuclear medium effects, and thus, the ratios $g_{A}^{\text {eff }} / g_{A}$ and $g_{V}^{\text {eff }} / g_{V}$ stand for the renormalization coefficients due to such correlations and nuclear medium effects that are not explicitly included in the nuclear model. Thus, they depend on the models and the transition operators. Since the axial-vector NMEs are strongly modified by the strong isobar (quark spin-isospin excitation) and other nonnucleonic correlations, we usually consider explicitly the renormalization coefficient $g_{A}^{\text {eff }}$, but the renormalization coefficient $g_{V}^{\text {eff }}$ for the vector coupling should be well considered unless the models include all correlations and the medium effect [6-8]. The DBD NMEs are discussed in review articles on neutrino nuclear responses [6-8]. Theoretical works on DBD NMEs are discussed in reviews [9-11].

The DBD rate $T_{r}^{0 v}$ per year (y) and per ton (t) of the DBD isotope-mass is expressed by using a neutrino mass unit $m_{0}$ as $[1,4,7,8]$

$$
\begin{gathered}
T_{r}^{0 v}=\left(\frac{m}{m_{0}}\right)^{2}, \quad m_{0}=\frac{m_{0}^{\prime}}{M^{0 v}}, \\
m_{0}^{\prime}=7.8 \mathrm{meV} \frac{A^{1 / 2}}{g_{A}^{2}\left(G^{0 v}\right)^{1 / 2}},
\end{gathered}
$$

where $A$ is the mass number of the DBD nucleus, $G^{0 v}$ is the phase space factor in a unit of $10^{-14} / \mathrm{y}$, and $m_{0}$ is the neutrino mass that gives the DBD rate $T_{r}^{0 v}=1$ per $\mathrm{t} y$. We define $m_{0}$ as the nuclear sensitivity of the DBD nucleus, being inversely proportional to $\left(G^{0 v}\right)^{1 / 2} M^{0 v}$. Here, high sensitivity to the neutrino mass means high capability of measuring a small neutrino mass. $m_{0}^{\prime}$ is the neutrino mass $m_{0}$ in case of $M^{0 v}=1$.

The values $m_{0}^{\prime}$ for typical DBD nuclei of ${ }^{82} \mathrm{Se},{ }^{100} \mathrm{Mo}$, ${ }^{116} \mathrm{Cd}$, and ${ }^{130} \mathrm{Te}$, which are of current interest, are all around $40 \mathrm{meV}$, which are close to the upper bound of the IH neutrino mass. The values for ${ }^{76} \mathrm{Ge}$ and ${ }^{150} \mathrm{Nd}$ are, respectively, larger and smaller by a factor 2 than the value of $40 \mathrm{meV}$ because of the smaller and larger phase space volumes.

In order to study the neutrino mass, the number of the $\mathrm{DBD}$ signals is required to be larger than the fluctuation 
$\delta$ of the number of the background signals. Then, it is expressed as $[1,7,8]$

$$
T_{r}^{0 v} \eta \varepsilon N T \geq \delta, \quad \delta=\delta_{0} \times(B N T)^{1 / 2},
$$

where $N$ is the total DBD isotope-mass in units of $\mathrm{t}, T$ is the exposure in units of $y, \delta_{0}$ is around $2, B$ is the number of the backgrounds per $N=1$ ton per $T=1$ year, $\eta$ is the enrichment coefficient of the DBD isotope, and $\varepsilon$ is the DBD signal detection efficiency. Note that the actual DBD isotope-mass is $\eta N$ ton, the signal yield is $T_{r}^{0 v} \eta \varepsilon N$ $T$, and the BG yield is $B N T$.

Then, the minimum effective neutrino mass to be measured with a $90 \%$ confidence level is expressed in terms of the nuclear sensitivity $m_{0}$ and the detector sensitivity $d$ as

$$
m_{m}=m_{0} \times d, \quad d=d_{0} \times \eta^{-1 / 2} \varepsilon^{-1 / 2}\left(\frac{N T}{B}\right)^{-1 / 4},
$$

where $d_{0}$ is around 1.4. The neutrino mass to be measured is $m_{m}=2 m_{0}^{\prime} / M^{0 v}$ in a typical case of the DBD detector exposure of $N T=1 \mathrm{t} y$, the BG rate of $B=1 / \mathrm{ty}, \varepsilon=0.55$, and $\eta=0.9$.

On the basis of the simple expression of the mass sensitivity $m_{m}$ as given in equation (5), key points for highsensitivity DBD experiments are as follows.

(1) The neutrino mass to be measured is proportional to $1 / M^{0 v}$ and $(B / N T)^{1 / 4}$. Therefore, the DBD nucleus with larger $M^{0 v}$ by a factor 2 is equivalent to the DBD isotope-mass $(N)$ larger by a factor 16 . Then, the detector volume gets more than an order of magnitude larger if $M^{0 v}$ gets smaller by $40 \%$

(2) The minimum mass to be measured depends on the ratio of $(B / N)^{1 / 4}$. Then, the neutrino mass sensitivity is improved by a factor 2 by increasing the DBD isotope-mass $(N)$ by a factor 16 , or by decreasing the $B G$ rate by a factor 16 , or by increasing the DBD isotope-mass by a factor 4 and decreasing the BG rate by a factor 4

(3) The effective neutrino mass depends on the neutrino mixing phases and is in the regions of $m \approx 17-45 \mathrm{meV}$ and $m \approx 1.5-4 \mathrm{meV}$, respectively, in cases of $\mathrm{IH}$ and $\mathrm{NH}$. Assuming a typical NME of $M^{0 v}=2$, one needs the DBD detectors with the mass sensitivities around the nuclear sensitivity of $m_{0} \approx 20 \mathrm{meV}$ and one-tenth of that, respectively, in cases of $\mathrm{IH}$ and $\mathrm{NH}$. Then, one needs DBD detectors with $N \approx 1 \mathrm{t}$ and $B \approx 1 / \mathrm{t} y$ and $N \approx 100 \mathrm{t}$ and $B \approx 0.01 / \mathrm{t} \mathrm{y}$, respectively, to measure the $\mathrm{IH}$ and $\mathrm{NH}$ neutrino masses

(4) DBD isotope enrichment is very effective. Enrichment by a factor 3 is equivalent to the increase of DBD isotope-mass by a factor 10 . Accordingly, it is effective to use ton scale $80-90 \%$ enriched DBD isotopes

\section{DBD Nuclear and Detector Sensitivities}

In this section, we discuss DBD nuclear and detector sensitivities as given in equation (5) for DBD experiments to access the $\mathrm{IH}$ and $\mathrm{NH}$ neutrino masses.

3.1. Nuclear Sensitivity. The nuclear sensitivity $m_{0}$ is a ratio of the unit mass $m_{0}^{\prime}$ and the NME $M^{0 v}$. In fact, $M^{0 v}$ is so sensitive to the details of the nuclear structures that accurate evaluation for $M^{0 v}$ is very hard. So, we discuss in the present work mainly $m_{0}^{\prime}$ and assume that $M^{0 v}$ is in the region of 13 (see the recent review on DBD NMEs [7] and references therein for detailed discussions on $M^{0 v}$ ).

The unit mass $m_{0}^{\prime}$ is proportional to $A^{1 / 2}\left(G^{0 v}\right)^{-1 / 2}$. The phase space factor $G^{0 v}$ increases with the increase of the DBD $Q$ value. Thus, double $\beta^{-}$nuclei with the large $Q$ around $3 \mathrm{MeV}$ are used. The large $Q$ is also effective to reduce much BGs from ${ }^{208} \mathrm{Tl}$ and ${ }^{214} \mathrm{Bi}$, which are two major BGs. The mass number $A$ dependence reflects the number of the DBD nuclei per ton of the total DBD isotope-mass. The mass sensitivities for a typical DBD nucleus with $m_{0}^{\prime}=40 \mathrm{meV}$ are plotted as a function of the exposure $N T$ in Figure 2. In order to access the IH mass of $20 \mathrm{meV}$, one needs a DBD exposure $N T$ of around $16 \mathrm{ty}$, i.e., $N \approx 5$ ton DBD isotopes and $T \approx 3 \mathrm{y}$ measurement in case of a typical NME of $M^{0 v}=2$ and BG rate of $B=1 / \mathrm{t} y$. On the other hand, one needs $N T$ of around 3 ty, i.e., $N \approx 1$ ton DBD isotopes and $T \approx 3$ y measurement in case of $M^{0 v}=3$, and $N T$ of around 250 ty, i.e., $N \approx 60$ ton DBD isotopes and $T \approx 4 \mathrm{y}$ measurement, in case of $M^{0 v}$ $=1$. So, it is very crucial to know the NME even for designing the DBD detector.

The mass sensitivities for the enrichment factors of $\eta=10$ , 50, and $100 \%$ are plotted as a function of the total DBD isotope-mass $N$ in Figure 3.

In order to access the IH mass of $20 \mathrm{meV}$, one needs $N$ $=3.4 \mathrm{t}$ and $4.1 \mathrm{t}$ of the total DBD isotopes, respectively, in case of $\eta=100 \%$ and $90 \%$, while it is $N=14 \mathrm{t}$ in case of $\eta=$ $50 \%(\eta N=7 \mathrm{t})$, and $N=340 \mathrm{t}$ in case of $\eta=10 \%(\eta N=34 \mathrm{t})$. Therefore, enrichment around $\eta \geq 80 \%$ is very effective. The 1-ton DBD total isotope-mass with $\eta \approx 90 \%$ is equivalent to the $N \approx 10$ ton of the total DBD isotope-mass with $\eta \approx 30 \%$.

3.2. DBD Detector Sensitivity and Backgrounds. The DBD experiment requires an ultra-low-BG detector since the DBD signal is very low in energy and the event rate is very rare. The neutrino mass sensitivity for a typical exposure time of $T=5 \mathrm{y}$ and $\eta=0.9, \varepsilon=0.55$, and $d_{0}=1.4$ is rewritten in terms of the ratio of the DBD isotope-mass $N$ and the BG rate $B$ as

$$
m_{m} \approx 1.35 \frac{m_{0}^{\prime}}{M^{0 v}(N / B)^{1 / 4}} .
$$

The mass sensitivities for the $m_{0}^{\prime}=40 \mathrm{meV}$ with typical NMEs of $M^{0 v}=2$ and $M^{0 v}=3$ are simply given as $m_{m} \approx 27$ $\mathrm{meV} /(N / B)^{1 / 4}$ and $m_{m} \approx 18 \mathrm{meV} /(N / B)^{1 / 4}$. They are shown as a function of $N / B$ in Figure 4. So, in the case of $M^{0 v} \approx 2$, DBD detectors to search for the IH mass of $20-30 \mathrm{meV}$ and 


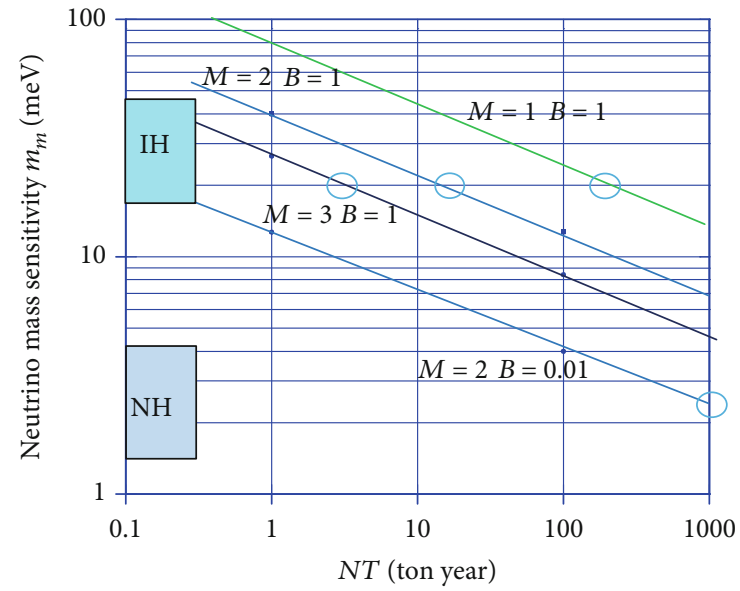

Figure 2: Neutrino mass sensitivities $m_{m}$ as a function of the exposure NT. $M$ and $B$ are the NME and the BG rate per ty. $d_{0}=$ $1.4, \varepsilon=0.55$, and $\eta=0.9$ (see equation (5)).

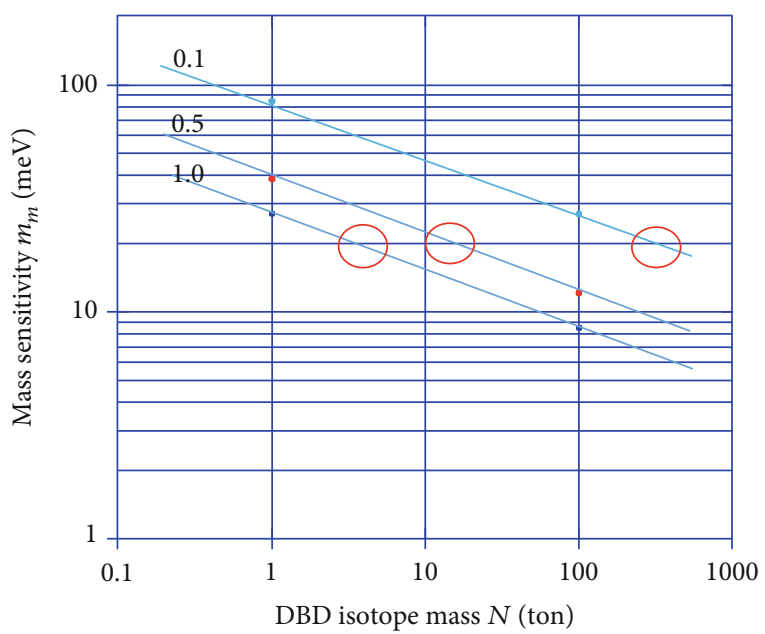

FIgURE 3: Neutrino mass sensitivities $m_{m}$ for $\eta=1.0,0.5$, and 0.1 as a function of the total isotope-mass $N$. The exposure time is $T=5 \mathrm{y}$, and the nuclear sensitivity of $m_{0}=20 \mathrm{meV}, \varepsilon=0.55, d_{0}=1.4$, and $B=1 / \mathrm{t} y$.

the $\mathrm{NH}$ mass of $2-3 \mathrm{meV}$ are required to be large isotopemass and low-BG detectors with $N / B \approx 1$ and $N / B \approx 10^{4}$, respectively.

Let us see how the mass sensitivity changes if one gets a $\mathrm{BG}$-free $(B=0)$ detector. In this case, the mass sensitivity is derived by requiring the signal yield $\geq 2.3$ as [4]

$$
m_{m}=1.5 \times m_{0} \varepsilon^{-1 / 2} \eta^{-1 / 2}(N T)^{-1 / 2} .
$$

The mass sensitivities for three BG cases of $B=1,0.01$, and 0 are compared in Figure 5. Then, the DBD isotopemass $N$ required to access the $\mathrm{IH}$ mass of $20 \mathrm{meV}$ is $1 \mathrm{t}$, while the DBD isotope-mass to access the $\mathrm{NH}$ mass of $2 \mathrm{meV}$ is $100 \mathrm{t}$. They are a factor 3 smaller than those for the detectors with $B=1$ and 0.01 . Thus, one needs 1 -ton scale and 100-ton scale DBD isotope-masses to access the $\mathrm{IH}$ and $\mathrm{NH}$ neutrino masses even by using the BG-free detectors.

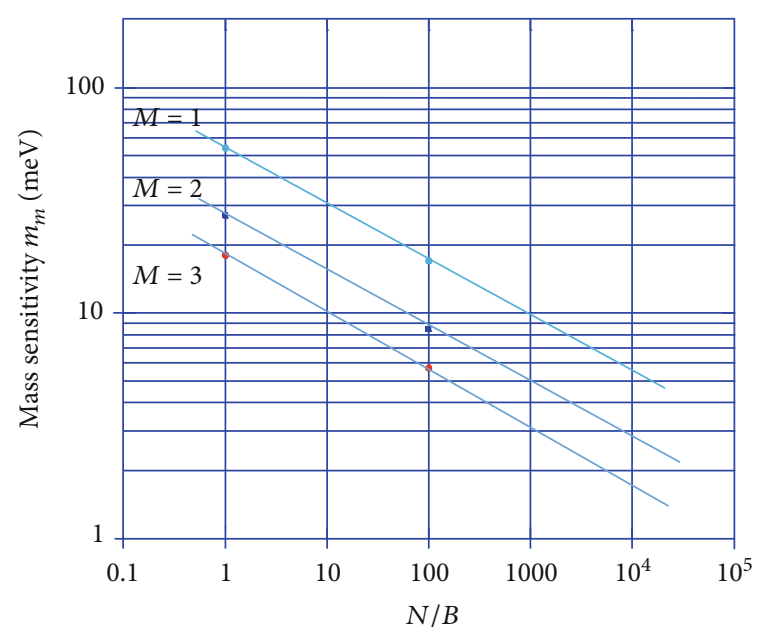

FIgURE 4: Neutrino mass sensitivities $m_{m}$ for $\eta=0.9, \varepsilon=0.55$, and $d_{0}=1.4$ as a function of the ratio of the total isotope-mass $N$ to the $\mathrm{BG}$ rate $B$. The exposure time is $T=5 \mathrm{y}$, and the unit mass $m_{0}^{\prime}=40 \mathrm{meV}$.

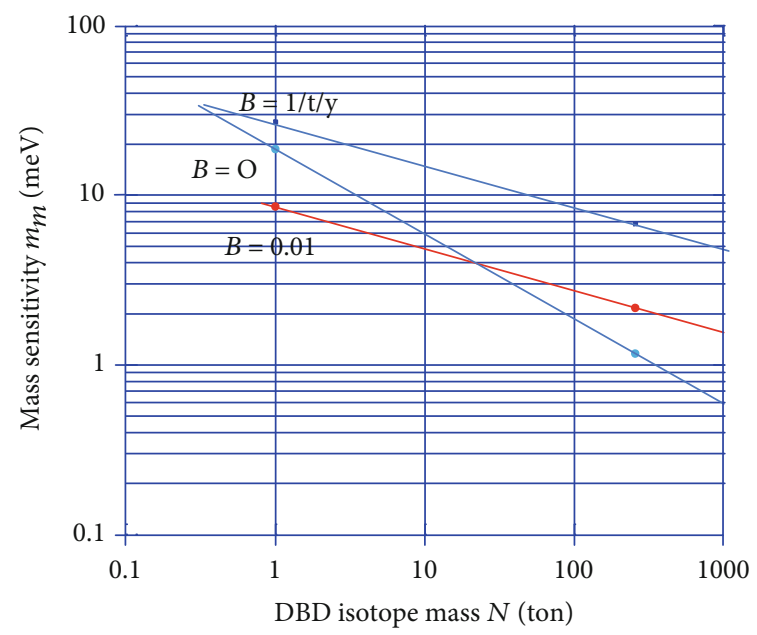

FIgURe 5: Neutrino mass sensitivities $m_{m}$ as a function of the DBD isotope-mass $N$ in cases of $B=1,0.01$, and 0 . The exposure time is $T=5 \mathrm{y}$. The unit mass $m_{0}^{\prime}=40 \mathrm{meV}$ and $M^{0 v}=2, \varepsilon=0.55$, and $\eta$ $=0.9$.

The DBD signal is expected to appear as a sharp peak at the energy of $E=Q$ in the energy spectrum. The peak width is around the FWHM (full width half maximum) of the detector. Thus, one usually sets the energy window of $\Delta E=$ 2 FWHM as the region of interest (ROI).

BGs in ROI are mostly due to $\beta$-rays and Comptonscattered $\gamma$-rays, which are continuum spectra. Backgrounds due to the solar neutrino interaction, which gets serious in the NH mass search, are also continuum $[12,13]$. Then, the $\mathrm{BG}$ rate at the energy window of $\mathrm{ROI}$ is proportional to FWHM. Since the mass sensitivity is proportional to $(B / N)^{1 / 4}$, improvement of the energy resolution by an order of magnitude is equivalent to an increase of the DBD isotope-mass by the same order of magnitude. 
The mass sensitivity depends on the detection efficiency as $m_{m}=k \varepsilon^{-1 / 2}$. The efficiency includes all efficiencies associated with the energy and PSA windows/cuts and analyses to select the DBD signals and to reject BG ones. It is around $\varepsilon$ $\approx 0.5-0.6$. Severe cuts to reject BGs reduce both the efficiency $\varepsilon$ and the BG rate $B$. The decrease of $\varepsilon$ by $20 \%$ is compensated if $B$ gets smaller by $40 \%$.

\section{Remarks and Discussions on DBD Detectors}

There are many high-sensitivity DBD experiments and future plans to access the IH mass around $20 \mathrm{meV}$ and the $\mathrm{NH}$ mass around $2 \mathrm{meV}$. The detailed reports on the present status of individual experiments and plans are given in their reports, and detailed discussions on them are given in the reviews $[1,4,5,7]$ and references therein and also in the recent Neutrino 2020 conference proceedings. The present and future DBD experiments are discussed there (see, for example, Ref. [14]). So, we give in this section a few comments on some (not all) possible DBD experiments from viewpoints of the DBD mass sensitivity, i.e., the nuclear and detector sensitivities as given in equation (5).

The DBD isotopes of ${ }^{82} \mathrm{Se},{ }^{100} \mathrm{Mo}$, and ${ }^{136} \mathrm{Xe}$ are very useful isotopes because of the small $m_{0}^{\prime} \approx 40 \mathrm{meV}$ due to the large phase space $G^{0 v}$ and the large $Q$ value and multiton scale enriched isotopes with $N \approx 10$ and $\eta \approx 90 \%$ are available by centrifugal separation.

Cryogenic ${ }^{82} \mathrm{Se}$ - and ${ }^{100} \mathrm{Mo}$ - bolometers with both thermal and scintillation signals are promising detectors with high-energy resolution and low-BG rate (see Refs. [15-18]). ${ }^{116} \mathrm{Cd}$ with $m_{0}^{\prime} \approx 40 \mathrm{meV}$ is also interesting (see Refs. $[19,20]$ ).

${ }^{136} \mathrm{Xe}$ can be easily enriched to get a $10 \mathrm{t}$ scale isotopemass and thus is possible to access the IH and NH masses. Various kinds of ${ }^{136} \mathrm{Xe}$ detectors are in progress (see Refs. [21-27]).

${ }^{76} \mathrm{Ge}$ with $Q=2.039 \mathrm{MeV}$ has the unit mass of $m_{0}^{\prime}=80$ $\mathrm{meV}$, a factor 2 larger than the others given above. Ton scale enriched DBD isotopes are possible by centrifugal separation, and the energy resolution of $10^{-3}$ in FWHM is very good. Accordingly, the Ge BG rate is almost an order of magnitude smaller than others to get the neutrino mass sensitivity comparable with other detectors (see Refs. [28-32]).

The natural abundance of ${ }^{130} \mathrm{Te}$ is $34 \%$, and thus, multiton scale natural Te isotopes may be used although the mass sensitivity $m_{m}$ gets larger by a factor 1.6 than the mass sensitivity with enriched ${ }^{130} \mathrm{Te}$-isotope detectors (see Refs. [18, 33]).

${ }^{150} \mathrm{Nd}$ has a large phase space factor because of the large $Q$ value of $3.4 \mathrm{MeV}$ and the large $Z$ number of 60 . Thus, $m_{0}^{\prime}=18 \mathrm{meV}$ is half of the others $[7,8]$. The natural abundance, however, is only $5.6 \%$, and enrichment is not easy (see Ref. [34]).

Tracking detectors have been used to study DBDs on ${ }^{100} \mathrm{Mo},{ }^{116} \mathrm{Cd},{ }^{82} \mathrm{Se}$, and many other DBD nuclei as discussed in review articles and references therein $[1,2,4,5]$. They measure individual two $\beta$-rays to identify the DBD signals and to reject single- $\beta$ background signals. The measured energy and angular correlations are used to differentiate the
DBD processes due to the left-handed and right-handed weak currents (see Refs. [35-37]).

Finally, it should be remarked that the NME is one of the key ingredients for the DBD mass sensitivity, and thus, selection of DBD isotopes with a large NME is very crucial for getting high-sensitivity DBD experiments [6,7]. It is however very hard to evaluate accurately the DBD NMEs. So, various experiments are in progress to provide nuclear parameters, the effective axial-vector weak couplings, and nuclear structures to help theoretical evaluations of the neutrinoless DBD NMEs. These are discussed in recent articles $[8,38]$.

\section{Data Availability}

There are no experimental data for this work because this is based on theoretical analyses and calculations.

\section{Conflicts of Interest}

The authors declare that they have no conflicts of interest.

\section{Acknowledgments}

The author thanks his colleagues for valuable discussions and RCNP Osaka University for the support and encouragement.

\section{References}

[1] H. Ejiri, "Double beta decays and neutrino masses," Journal of the Physical Society of Japan, vol. 74, no. 8, pp. 2101-2127, 2005.

[2] F. T. Avignone, S. R. Elliott, and J. Engel, "Double beta decay, Majorana neutrinos, and neutrino mass," Reviews of Modern Physics, vol. 80, no. 2, pp. 481-516, 2008.

[3] H. Ejiri, "Double $\beta$-decays and neutrino nuclear responses," Progress in Particle and Nuclear Physics, vol. 64, no. 2, pp. 57-249, 2010.

[4] J. D. Vergados, H. Ejiri, and F. Šimkovic, "Theory of neutrinoless double-beta decay," Reports on Progress in Physics, vol. 75, no. 10, article 106301, 2012.

[5] J. D. Vergados, H. Ejiri, and F. Šimkovic, "Neutrinoless double beta decay and neutrino mass," International Journal of Modern Physics E, vol. 25, no. 11, article 1630007, 2016.

[6] H. Ejiri, "Nuclear spin isospin responses for low-energy neutrinos," Physics Reports, vol. 338, no. 3, pp. 265-351, 2000.

[7] H. Ejiri, J. Suhonen, and K. Zuber, "Neutrino-nuclear responses for astro-neutrinos, single beta decays and double beta decays," Physics Reports, vol. 797, pp. 1-102, 2019.

[8] H. Ejiri, "Neutrino-mass sensitivity and nuclear matrix element for neutrinoless double beta decay," Universe, vol. 6, no. 12 , p. 225, 2020.

[9] J. Suhonen and O. Civitarese, "Weak-interaction and nuclearstructure aspects of nuclear double beta decay," Physics Reports, vol. 300, no. 3-4, pp. 123-214, 1998.

[10] J. Suhonen and O. Civitarese, "Double-beta-decay nuclear matrix elements in the QRPA framework," Journal of Physics G: Nuclear and Particle Physics, vol. 39, no. 8, article 085105, 2012.

[11] J. Engel and J. Menéndez, "Status and future of nuclear matrix elements for neutrinoless double $\beta$-decay: a review," Reports on Progress in Physics, vol. 60, article 046301, 2017. 
[12] H. Ejiri and S. R. Elliott, "Charged current neutrino cross section for solar neutrinos, and background to $\beta \beta(0 v)$ experiments," Physical Review C, vol. 89, no. 5, article 055501, 2014.

[13] H. Ejiri and S. R. Elliott, "Solar neutrino interactions with the double- $\beta$ decay nuclei ${ }^{82} \mathrm{Se},{ }^{100} \mathrm{Mo}$, and ${ }^{150} \mathrm{Nd}$," Physical Review C, vol. 95, no. 5, article 055501, 2017.

[14] J. Detwiler, "Future neutrino-less double-beta decay experiments," Neutrino, vol. 2020, 2020.

[15] D. R. Artusa, A. Balzoni, J. W. Beeman et al., "The LUCIFER/CUPID-0 demonstrator: searching for the neutrinoless double-beta decay with $\mathrm{Zn}^{82}$ Se scintillating bolometers," Journal of Physics: Conference Series, vol. 888, article 012077, 2017.

[16] H. Park and AMoRE collaboration, "The AMoRE: search for neutrinoless double beta decay in ${ }^{100} \mathrm{Mo}$," Nuclear and Particle Physics Proceedings, vol. 273, p. 2630, 2016.

[17] D. Poda and A. Giuliani, "Low background techniques in bolometers for double-beta decay search," International Journal of Modern Physics A, vol. 32, no. 30, article 1743012, 2017.

[18] T. O. Donnell, "CUORE results and CUPID Project," Neutrino, vol. 2020, 2020.

[19] K. Zuber, "COBRA-double beta decay searches using CdTe detectors," Physics Letters B, vol. 519, no. 1-2, pp. 1-7, 2001.

[20] F. A. Danevich, A. S. Barabash, P. Belli et al., "Search for double beta decay of ${ }^{116} \mathrm{Cd}$ with enriched ${ }^{116} \mathrm{CdWO}_{4}$ crystal scintillators," Journal of Physics: Conference Series, vol. 718, article 062009, 2016.

[21] A. Gando, Y. Gando, T. Hachiya et al., "Search for Majorana neutrinos near the inverted mass hierarchy region with KamLAND-Zen," Physical Review Letters, vol. 117, article 082503, 2016.

[22] J. B. Albert, G. Anton, I. Badhrees et al., "Search for neutrinoless double- $\beta$-decay with the upgraded EXO-200 detector," Physical Review Letters, vol. 120, article 072701, 2018.

[23] L. Yang and EXO-200 and nEXO Collaborations, "Status and prospects for the EXO-200 and nEXO experiments," Journal of Physics: Conference Series, vol. 888, article 012032, 2017.

[24] G. Anton, I. Badhrees, P. S. Barbeau et al., "Search for neutrinoless double- $\beta$ decay with the complete EXO-200 data set," Physical Review Letters, vol. 123, article 161802, 2019.

[25] C. Grant, "Results from KamLAND-ZEN and SON+," Neutrino, vol. 2020, 2020.

[26] J. J. G. Cadenas, "Xe-136 experiments, present and future," Neutrino, vol. 2020, 2020.

[27] N. L. March, "The NEXT high pressure xenon gas TPC for neutrinoless double $\beta$-decay searches," Journal of Instrumentation, vol. 13, article C01048, 2018.

[28] M. Agostini, A. M. Bakalyarov, M. Balata et al., "Improved limit on neutrinoless double $\beta$-decay of ${ }^{76} \mathrm{Ge}$ from GERDA Phase II," Physical Review Letters, vol. 120, article 132503, 2018.

[29] C. E. Aalseth, N. Abgrall, E. Aguayo et al., "Search for neutrinoless double- $\beta$ decay in ${ }^{76} \mathrm{Ge}$ with the Majorana demonstrator," Physical Review Letters, vol. 120, article 132502, 2018.

[30] S. I. Alvis and Majorana collaboration, "Search for neutrinoless double- $\beta$ decay in ${ }^{76} \mathrm{Ge}$ with $26 \mathrm{kgy}$ of exposure from the Majorana demonstrator," Physical Review C, vol. 100, article 025501, 2019.

[31] N. Abgrall, A. Abramov, N. Abrosimov et al., "The large enriched germanium experiment for neutrinoless double beta decay (LEGEND)," AIP Conference Proceedings, vol. 1894, article 020027, 2017.
[32] Y. Kermaidic, "GERDA, Majorana and LEGEND towards a background-free ton-scale Ge76 experiment," Neutrino, vol. 2020, 2020.

[33] D. Q. Adams, C. Alduino, K. Alfonso et al., "Improved limit on neutrinoless double-beta decay in ${ }^{130} \mathrm{Te}$ with CUORE," Physical Review Letters, vol. 124, article 122501, 2019.

[34] S. Andringa, E. Arushanova, S. Asahi et al., "Current status and future prospects of the SNO+ experiment," Advances in High Energy Physics, vol. 2016, Article ID 6194250, 2016.

[35] H. Ejiri, J. Engel, R. Hazama, P. Kurastev, N. Kudomi, and R. G. H. Robertson, "Spectroscopy of double-beta decays from ${ }^{100}$ Mo for neutrinos," Physical Review Letters, vol. 85, p. 2917, 2000.

[36] H. Ejiri, P. Doe, S. R. Elliott et al., "MOON (Molybdenum Observatory Of Neutrinos) for neutrinoless double $\beta$ -decays," European Physical Journal, vol. 162, p. 239, 2008.

[37] D. Waters and NEMO-3 and SuperNEMO Collaborations, "Latest results from NEMO-3 and status of the superNEMO experiment," Journal of Physics: Conference Series, vol. 888, article 012033, 2017.

[38] H. Ejiri, "Perspectives on neutrino nuclear-response studies for double beta decays and astro neutrinos," Frontiers in Physics, 2021. 